\section{CASE OF EXTRA-UTERINE GESTATION.}

\section{By C. E. GOOdING, M.D. McGitil Coli., M.R.C.S.}

UNFORTUNATELY cases of extra-uterine gestation do not occur so rarely as perhaps to warrant a single instance being put on record by itself. It is only on account of some specially interesting features which it presented that $I$ venture to report the following case.

Early in December, 1885, I was called to a Quadroon woman, aged forty-nine and unmarried. She was suffering from well-marked symptoms of acute cystitis, following apparently on exposure to cold and damp a few days before. She also complained of a constant and severe pain, with great tenderness in the right iliac region, shooting thence down the thigh of the same side. Some fulness and diminished resonance on percussion could be detected in the right iliac fossa, and extending slightly beyond the middle line of the abdomen. Conjoined vaginal examination revealed a hard, non-fluctuating, and very sensitive swelling, about the sizg of a coconut, rising out of the pelvis on the right side. The uterus, which was of normal depth, was pushed over to the left, but moved separately from the tumour. The latter was diagnosed as ovarian in its origin, and from its density probably fibroid or dermoid. Under appropriate treatment the vesical symptoms entirely subsided in a couple of weeks, and the patient was then lost sight of for some time, although told to report herself, should other symptoms due to the presence of the tumour develop. Thirteen months after, in January, 1887, she was admitted under my care in the St. Philip's Almshouse. She brought with her three bodies slightly encrusted with phosphates, which she had passed per urethram during the previous week, and which were at once recognised as the tibia and two ribs of a child apparently at or near full term. I learnt then that since I had seen her she had had several attacks of cystitis, and been under the care of more than one medical man, with but very partial relief to her symptoms. The following history was now elicited : Began to menstruate at fourteen, and was regular until twenty, when her first and only child was born. With the exception of very slight occasional intermissions, she continued regular until ten years ago. Her menses then ceased for five or six months, but although she had exposed herself to the chances of conception she did not consider herself pregnent. She declares positively that she did not observe any of the usual signs of pregnancy, but admits that she was unusually fat about this period. Hrom this time, however, she has never felt strong, being easily tired and very subject to pains in the back and lower part of abdomen, and running down the leg on the right side. After this interruption her menses returned and were fairly regular until they ceased entirely three years ago.

The woman's condition on admission was as follows:She has become very much emaciated since last seen; is suffering with chronic cystitis; micturition very frequent, and the act accompanied with intense pain; bladder when sounded is found to contain a large number of bones; dulness over tumour has lessened in extent, and tumour itself per vaginam feels more soft and yielding; is suffering with hectic, and has an evening temperature in the neighbourhood of $101^{\circ}$

She was ordered tonics and good diet, and the bladder to be washed out frequently with antiseptic injections. Under this treatment her general condition improved, and after two or three wesks (during which time she had passed a couple more small bones), on consultation with Dr. Archer I decided to operate. This was done on Februsry 12tb, Dr. Archer assisting; Dr. Greaves administered ether, and Dr. Shepherd also was present. No injection was used to distend the bladder on account of the manifest communication existing between that viscus and the fotal cyst. The urethra was first dilated until it admitted the index finger, and the bladder was then opened by an incision in the median line above the pubes. With the two index fingers-one passed through the urethra, and the other through the abdominal wound-all the bones were removed through the latter. The bones were found partly in the bladder and partly in a cyst--about the size of the foetal head at term, - which communicated freely with the fundus of the bladder on the right side through a fistulous channel large enough to easily admit the finger. The bladder and cyst were next washed out thoroughly with a full stream of warm carbolised water in order to remove any remaining detritus. The opening into the bladder was left unsuturea, and that night all the urine passed through it.

The next morning a catheter was tied into the bladder through the urethra and retained for one day. After this all the urine passed per urethram, though it was several weeks before complete control over the sphincter vesic was established. After the operation there were no bad symptoms. The bladder was washed out with antiseptic injections untrl its coats had regained their normal condition. In six weoks the abdominal opening had healed all but a little fistula at the upper end, through which a probs could be passed directly backwards for about four inches. A small bit of loose bone was now detected near the bottom, and its removal was soon followed by the healing of the sinus. At the end of three months she was discharged, "feeling better and stronger," as she expressed it, " than she had for many years."

The majority of all cases of extra-uterine gestation end in the death of the mother through rupture of the cyst and consequent hæmorrhage, which usually takes place about the third or fourth month. Sometimes the foetus dies in the early months of pregnancy without rupture of the cyst; then the fluid parts are absorbed and the remainder dries up and becomes quiescent. In a smaller proportion of cases the foetus does not perish until the full term of gestation, and then the ultimate result varies greatly. The fiuid contents of the cyst may dry up, and the foetus (bocoming converted into a substance resembling adipocere) remain quiescent for an indefinite time. On the other hand, the cyst with its contents may undergo suppuration, and the mother die from the violence of the inflammatory process. Should, however, the course of inflammation be less intense, an effort is made to throw off the fotus (which has now become a foreign body) by the formation of a fistulous canal leading from the cyst. This most frequently opens into some part of the intestines, of through the walls of the abdomen, or more rarely into the vagina or bladder. The case recorded above is remarkable, as affording an example of that exceedingly rare termination of an extra-uterine pregnancy-viz., an attempt on the part of nature to effect a cure by getting rid of the contents of the cyst through a fistula into the bladder. It is also interesting from the slight amount of constitutional diso turbance with which the growth of the fotus in its unusual position was accompanied, and from the entire want of those subjective and objective signs of pregnancy, the absence of which prevented the woman and those about her from entertaining the slightest suspi ion of her being pregnant.

\section{THE TREATMENT OF CYSTIC GOITRE. ${ }^{1}$}

BY T. MARK HOVELL, F.R.C.S. EDIN.,

SENIOR SURGEON AND AURAL SURGEON TO THE HOSPITAT FOR DISEASES OF THE THROAT; JUNIOR AURAL SURGEON TO THE LONDOX

HOSPITAL; LECTURER ON DIESASES OF THE THROAT

AT THE LONDON HOSPITAL MEDICAL COLLEGE.

Mr reason for bringing forward a method of treatment by no means new is that, although it is mentioned in all the principal English and American works on Surgery, the prominence it deserves is not accorded to it, and recently cases have been published in which the treatment adoptêd has entailed serious risk to the patient, whose safety would not have been thus unnecessarily jeopardised had the measures I am about to describe been adopted.

The treatment which I advocated is that introduced by Sir Morell Mackenzie in a paper read before the Hunterian Society on Jan. 22nd, 1872, and published by him in THE LANCET of May 11th, 1872 (vol. i., p. 642). ${ }^{2}$ His method briefly consists in tapping the cyst, and when the fluid has been withdrawn injecting a solution of perchloride of iron through the cannula of a syringe and allowing it to remain for a certain time, so as to convert the cyst into a chronic abscess It is essential that the trocar should be introduced at the most dependent part of the tumour to ensure subsequently efficient drainage, upon which greatly depends the success of the operation, and it is an advantage to make the puncture where the cyst wall is thin, provided this condition is found in the position mentioned. Care must be taken to avoid a place traversed by a large vein, and when practicable it is well to introduce the trocar as near the mesial line as possible. The spot having been selected, the skin should

1 Read before the Hunterian Society, April 13th, 1887.
2 See also Birmingham Medical Review, Fol. iv., 1875. 
now be frozen by means of an ether spray, and when anæsthesia has thus been produced, a trocar with silver cannula attached should be passed through the wall of the cyst, the trocar being withdrawn as soon as the cavity is reached, and thecannula pushed furtherin by means of a blunt-pointed plug. For a large goitre I select a cannula two inches in length, because I have found that if it is shorter it is apt to slip out of the cyst, in which case there is of ten difficulty in reintroducing it by means of the plug, in consequence either of the cyst wall contracting and thereby causing the hole on it to be no longer opposite the opening in the skin, or from the aperture in the cyst wall having closed. When this occurs it is necessary to again use the trocar. For smaller goitres a cannula of porportionate length should be employed. It is not necessary to use a very large cannula ; the sizes I employ correspond to Nos. 7, 8, and 9, English catheter gauge. Those smaller than No. 7 are apt to become blocked with discharge, whereas those larger than No. 9 are unnecessary in ordinary cases; should a larger cannula be required during the subsequent treatment of a case, it can easily be introduced. The cannula having been inserted into the cyst, it should be secured in position by a tape passed round the neck. The plug should be then withdrawn and the contents of the cyst allowed to escape, care being taken not to squeeze the cyst lest air should be sucked into its cavity. One or two drachms or more, according to the size of the cyst, of a solution of perchloride of iron (two drachms of the salt to an ounce of water) should be now injected into the cavity by means of the syringe just described, held vertically, and the plug reintroduced to prevent the solution from escaping. The reasons for using the aqueous solution of the salt as mentioned are that it is more astringent than either the incture or liquor ferri perchloridi (B.P.), and it is devoid of the free hydrochloric acid which they contain. The syringe ${ }^{3}$ used for this purpose has its nozzle fixed at an obtuse angle with the cylinder, the object of this arrangement being to allow any air which may be in the syringe, when held vertically, to rise to the top of the solution, and therefore render impossible the injection of air into a vein. This occurrence is further prevented by a button placed on the piston of the syringe above the cylinder, the button being screwed down for about an inch after the cylinder has been filled with the solution for injection, causing, by this means, the piston to be about an inch shorter than the cylinder, and consequently incapable of emptying it, The nozzle of the syringe is made to taper to the point in order to allow it to accurately fit any cannula which may be used for this operation. The solution of iron having been injected into the cyst, it should be allowed to remain there for seventytwo hours. During this period the cyst will refill to a greater or less extent, and if inflammation has been set up, it will be marked by pain and tenderness over the tumour, and there may, in addition, be signs of slight inflammation in its immediate neighbourhood. Whilst the cyst is swelling care must be taken to loosen the tape which holds the cannula to a corresponding degree, otherwise the head of the cannula will be pressed against the skin and may cause it to slough; the tight tape will also greatly add to the patient's discomfort. During the after-treatment of the case, tightness of the tape may generally be considered to indicate distension of the cavity of the cyst consequent upon the escape of pus being retarded by the cannula having become obstructed. If on withdrawing the fluid at the end of seventy-two hours it is found to contain blood or to show no signs of suppuration, an injection of iron must again be made and retained in the sac for seventy-two hours as before by means of the plug, this process being repeated until suppuration has been produced. Poultices should then be applied, and in some cases the plug may be permanently removed, but usually it is better to keep it in position for a few days in order to retain some pus in the cvst and increase thereby the amount of inflammation. The quantity of pus, however, which is retained should not be large, and to ensure this the plug must be removed several times during the day, to allow the discharge which has collected to escape. Whilst the plug is retained in the cannula the temperature generally ranges between $100^{\circ}$ and $10 g^{2}=\mathrm{F}$, but quickly falls after the plug has been removed and the free exit of pus permitted; but should it not do so (and from the character of the discharge it appears unnecessary to keep up the inflammation), the cyst should be washed out several times a day by the injection through the cannula of tepid water containing some antiseptic.

Throughout the subsequent treatment of the case it is most important that thorough drainage of the cyst should be maintained; and it should be constantly borne in mind that pyrexia persisting after the plag has been removed from the cannula, or a sudden elevation of temperature during the later stages of the case, is almost invariably due to the cannula being blocked, and consequently causing retention of pus in the cavity of the cyst. With the view of preventing this occurrence I have for some years past employed a piece of Ellis's drainage tube, which consists of a piece of silver or plated wire wound spirally to form a tube. I usually allow the turns of wire to remain touching one another, but in some cases it is well to slightly separate them, which is easily done by making gentle traction. To apply it, I select a piece of tubing which is a size smaller than the cannula, and consequently passes easily through it; and having ascertained the precise length of the cannula by measuring the plug belonging to it, I introduce the tubing until it projects about half an inch beyond it into the cyst, and then secure it in that position by fastening its other end to the tape around the patient's neck. By this means although the more solid portions of the discharge gathers round the cannula and the piece of tubing which projects from it and there remains, the fluid portion readily passes between the coils of wire into the lumen of the tube and thence away. To further ensure efficient drainage it is necessary to wash out the cyst with tepid water, to which an antiseptic may be added several times a day, and before doing so to remuve the Ellis's tubing. In some cases the mere injection of water into the cyst is not sufficient to entirely remore all the thicker portion of the discharge, which, carried by the outward flow of the water, collects around the inner opening of the cannula and blocks it. When this occurs, a fine probe introduced through the cannula as soon as the syringe is withdrawn, and moved about so as to keep the end of the tube clear, will cause the previously obstructing pieces to come away with the water. It is useless to attempt to employ Ellis's tubing without a cannula, or to leave it long in position without being removed, because the granulations grow between the coils of wire and choke the lumen of its tube. Care must be taken to bend the inner cut extremity of the wire so that it does not prick or scratch the wall of the cyst. Constant retention of a certain amount of pus may be caused by the cannula projecting too far into the cyst, and consequently only allowing the escape of the overflow; the existence or not of this state of affairs can be easily determined by removing the cannula and noticing its colour, the portion which projects into the cyst being always more darkly stained than that which is in contact with the tissues.

It sometimes happens that after a few weeks the discharge becomes thin; and no appreciable diminution in the size of the cyst takes place from week to week. This condition is usually produced by want of tone on the part of the patient, causing the granulations to become flabby and the healing process generally less active. To remedy this, in addition to tonics and a more generous diet, it is well after each time the cyst is washed out to inject a solution of chloride of zinc (fifteen to thirty grains to an ounce of water) into the cyst, and allow it to escape through the cannula, and to continue to do so until, from the ready way in which the walls bleed when touched with a probe and other indications, it is seen that the granulations bave reassumed a healthy aspect. A cannula should be retained in the cyst until its cavity is completely obliterated. Provided that efficient drainage is obtained through the cannula which was at first introduced, it is a mistake to remove it until the cyst has contracted around its inner extremity and the granulations have begun to block up the aperture. When this takes place the cannula should be replaced by one of the same gauge, but not more than a quarter of an inch shorter, which should likewise be retained until the granulations begin to obstruct its inner orifice, when it should be replaced by a cannula again not more than a quarter of an inch shorter than the former one. A cannula should be introduced on this system until the sinus made by the first cannula becomes too short to retain it, when the wound may be left to granulate. Sir Morell Mackenzie sometimes uses a silver cannula with a movable shield, which can be fixed at any part by means of a screw. In this way as the cyst contracts the tube can be shortened, and its length accurately adjusted to the requirements of the case. For patients who are not well off it is convenient, because it renders a number of tubes un- 
necessary; but it has the disadrantage of projecting further in front of the neck in proportion as it is withdrawn from the cyst, and this causes a little inconvenience in the arrangement of the dress and the application of poultices. A multilocular cyst should be treated in the same way as a simple cyst; but if it is found that pus is retained in any

Fig. 1.

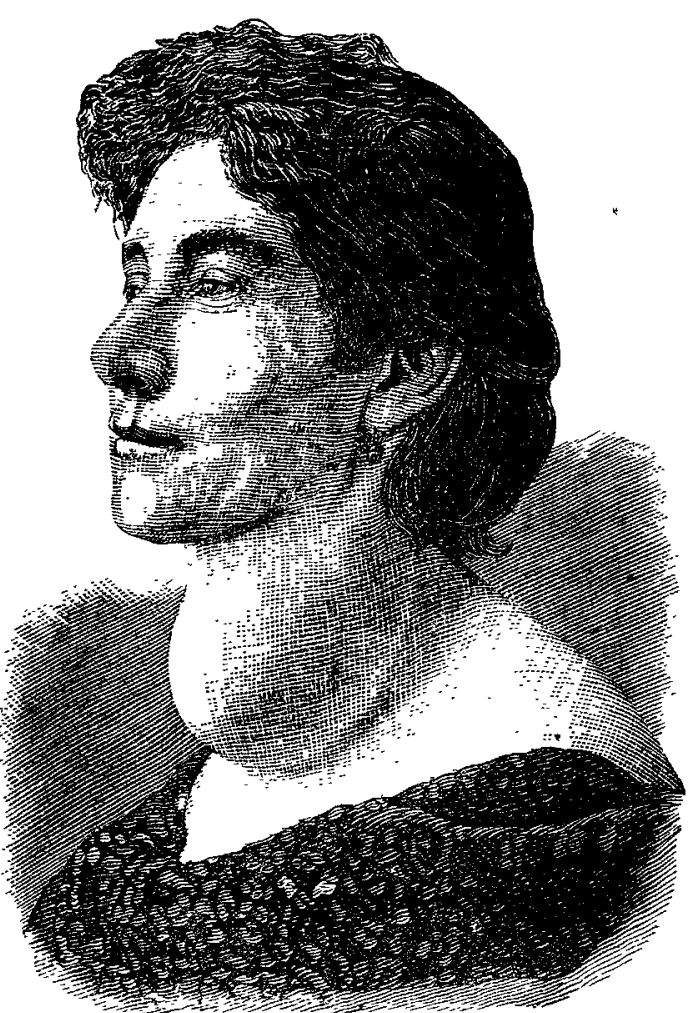

one of the cavities, a cannula must be passed directly into that cavity at its most dependent part, and the same treatment adopted for it as is employed in the case of a simple cyst. A cyst containing a large amount of calcareous matter in its walls can only be effectually treated by excision; but this operation ought not to be performed

FIG. 3.

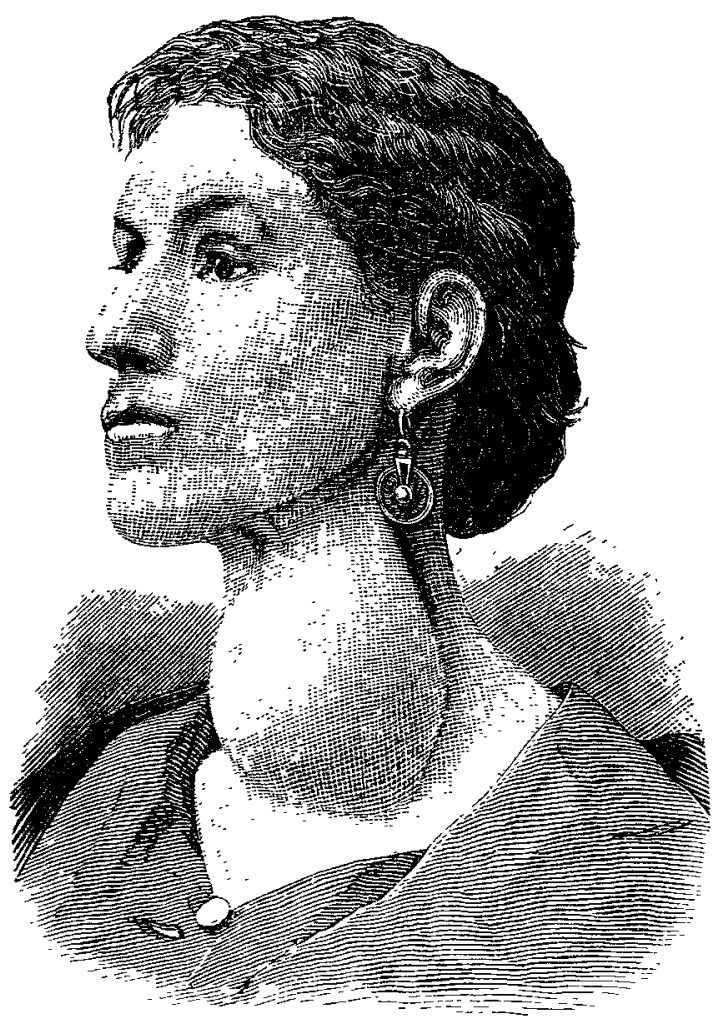

unless the symptoms produced by the tumour demand surgical interference.

The following cases illustrate the mode of treatment which I have described. The accompanying woodcuts faithfully represent the appearance of the patients before and after treatment.
$\mathrm{CASH}_{\mathrm{A}}$ 1.-Marie $\mathrm{K}-\cdots$, aged thirty-four, single, first noticed a swelling in her neck twenty years ago. During the last eight years she has had a certain amount of dyspncea whilst walking fast or going upstairs. Her voice first became weak five or six years ago, and during the last four years it has remained much in its present condition.

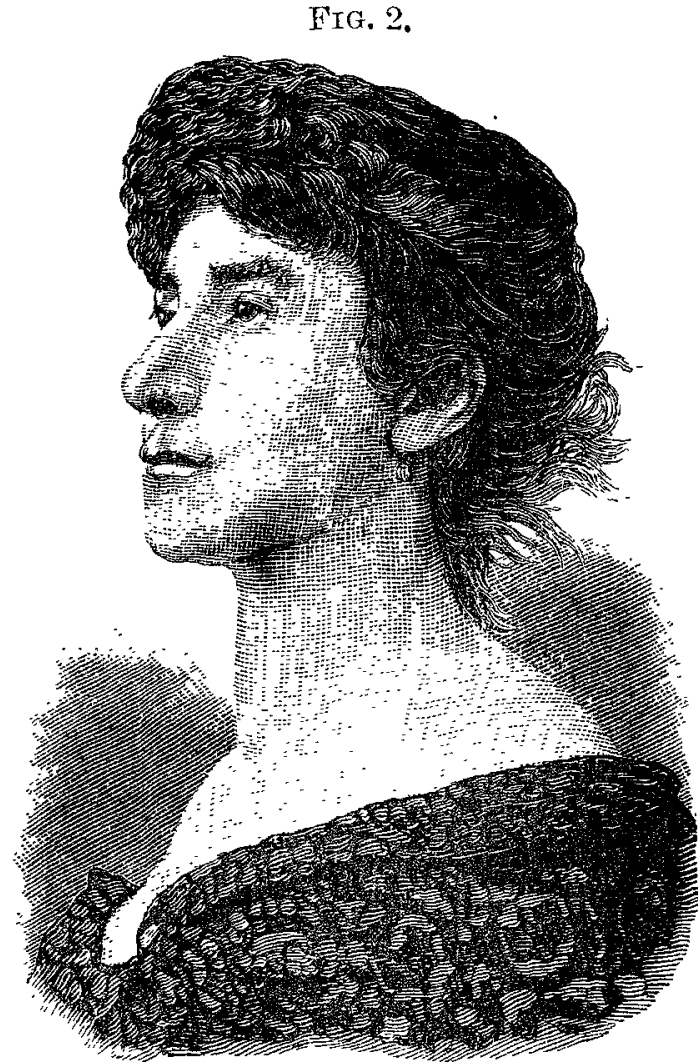

There is now paralysis of the left vocal cord, which is in the cadaveric position. The tumour has increased very much in size during the last three years, so much so that for this period she has been unable to wear a collar. The goitre had never been surgically treated before she came to the Throat Hospital. On admiesion the circumference of

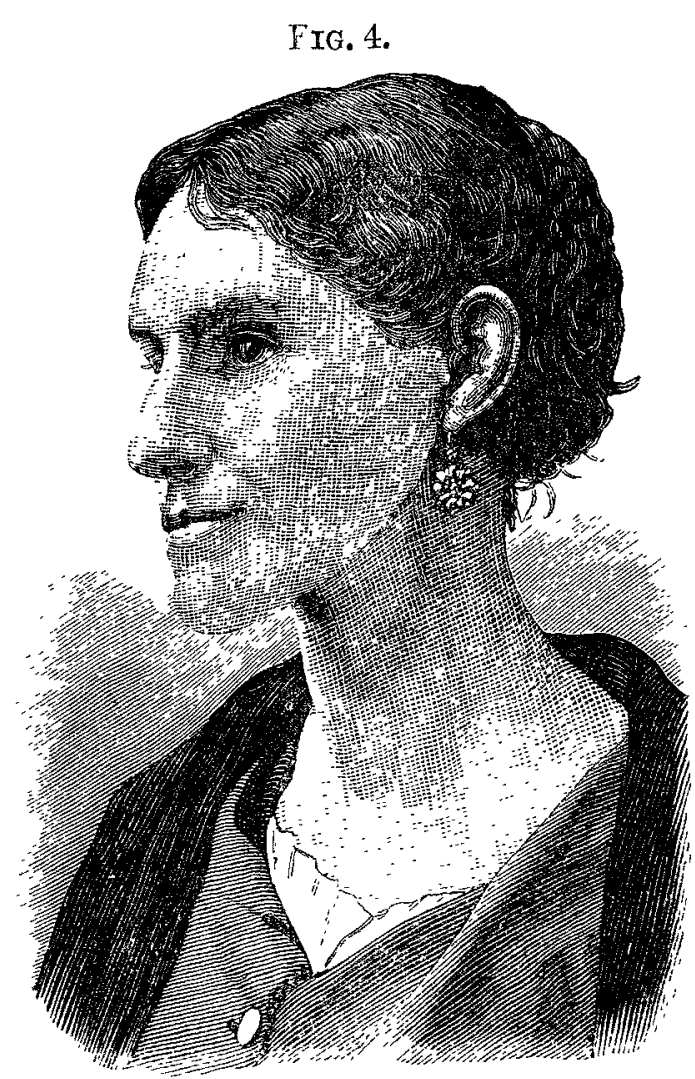

her neck measured nineteen inches. The cyst was tapped on Dec. 16th, 1886, and twenty-one ounces of dark-brown fluid withdrawn. (See Figs. 1 and 2.)

CASE 2.- Mrs. C-, aged thirty-three, first noticed the enlargement in her neck nineteen years ago; the goitre was then about the size of a hen's egg. For several years it 
did not increase much in size. About six years ago the swelling began to enlarge, and continued to increase slowly. She married three years ago, and was confined ten months afterwards, a marked increase in the size of the tumour being noticed after the birth of the child. Mrs. C- - states that during the last twelve months when she has run downstairs she has felt the fluid moving about " like water in a bottle." Durng the three months previously to her admission into the Throat Hospital the increase in size was very great, so much so that she was obliged to enlarge the collar of her dress almost every Saturday in order to enable her to wear the dress on the Sunday. The cyst was tapped on Jan. 31st, 1887 , and ten ounces and a half of bright-red fluid withdrawn. (See Figs. 3 and 4 .)

Having described Sir Morell Mackenzie's method, it may not be out of place to take a glance at the various other plans of dealing with cystic goitre which have been tried or recommended.

In TeE LANCET of July $3 r d, 1886$, is an account of a cystic goitre treated by Dr. C. T. Vachell of Cardiff by "shelling out." The patient was a woman aged twenty-three, who had a tumour about the size of a cocoanut occupying the position of the right lobe of the thyroid gland and passing somewhat over the middle line of the neck. Fluctuation could be felt all over the tumour. About three weeks before admission a teacupful of bloody serous fluid was withdrawn by a trocar and cannula, and the tumour was considerably reduced in size, butin less than six weeks the tumour had attained a larger size than before, the circumference of the neck being

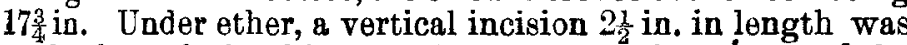
made through the skin over the most prominent part of the tumour and at once the surface of it came into view. "Up to this point very little bæmorrhage had occurred. Without much difficulty the tumour was now shelled out, but the hæmorrhage which commenced with this process and continued thronghout was very profuse. Firm pressure was maintained by an assistant, the surface was mopped with very hot water mixed with tincture of iodine ( 1 in 20), and as bit by bit the surface was exposed all the bleeding points were ligatured. The edges were then brought together and a drainage tube inserted. The tumour, which was irregularly oroid in shape, measured $13 \mathrm{in.}$ by $9 \mathrm{in}$ and weighed twenty ounces. On being cut into nine ounces of dark sanguineous fluid escaped similar to what had been removed by tapping, but darker." The patient left the infirmary twelve days after the operation.

Although the operation was most skilfully performed, and the result of the treatment as far as reported quite successful, still I consider from the account given of the case that there was nothing to justify the measures which were adopted. The case was apparently an ordinary one of cystic goitre, and it is distinctly stated that neither breathing nor swallowing was seriously interfered with. That the cyst would refill after being tapped might have been foretold, it being the exception when this does not occur; also that it would be liable to attain a greater size might have been stated with equal confidence. Tbese usual results, however, having followed the tapping, Dr. Vachell appears to have thought that no course was open to him but to excise the portion of the gland containing the cyst. Although in the case just recorded the operation was successful, its performance is always attended with considerable amount of risk to the patient. More than one death has resulted from hæmorrhage, which, as it was in this case, is frequently very profuse, and the evil affects which may arise from excision of large portions of the thyroid gland are too well known to need comment. After the grave consequences which are liable to attend the operation, the mere question of the size of the scar which results sinks into insignificance, although I hold that in the surgical treatment of exposed portions of the body this point ought to be considered.

In THF LANCET of Jan. 22nd, 1887, p. 174, is the report of a paper which Mr. Mayo Robson of Leeds, read before the Cinical Society, in which he advocated the treatment of thyroid cysts by antiseptic incision, stitching the edge of the cyst to the skin and scraping the interior of the cyst sufficiently to sepsrate all colloid material, then draining For a short time under an untiseptic dressing, and afterwards packing with zinc lotion and lint. Mr. Mayo Robson thought that the advantages of this method were its simplicity, safety, and certainty. He stated that he would not again practise complete excision, having had one case in which asphyxia and another in which hæmorrhage caused great anxiety. The advantage claimed by Mr. Mayo Robson of stitching the cyst wall to the skin was that by this means the cyst was shut off from the surrounding cellular tissue, and consequently the extension of inflammatory processes was prevented.

The elaboration of the treatment by incision just referred to presents no novel feature, as the following extract from Gross's "Systum of Surgery" (1882, vol. ii., p. 356) clearly shows: "Incision of the sac and uniting its walls with those of the skin, an operation which I believe originated with von Bruns, has been performed eleven times by Billroth, with three deaths." The fact that three deaths occurred in the eleven cases operated upon by Billroth does not offer much encouragement for the adoption of this method of treatment, especially if he did not scrape the interior of the sac, which proceeding must add considerably to the risks of it. As I consider that the whole operation recommended by Mr. Mayo Robson is open to serious objection, I need not dwell upon this refinement of it, further than to remark that the advantages claimed by the plan proposed are open to question, and to endorse the statement made by Mr. H. H. Clutton, that a sinus is liable to result from the course advocated. The object of the treatment advised by Mr. Mayo Robson is the same as that of Sir Morell Mackenzieviz., destruction of the secreting surface of the cyst and subsequent union of its walls; but the means taken to produce this result compare most unfavourably with those instituted by Sir Morell Mackenzie.

Before the secreting surface can be destroyed it must be reached. Mr. Mayo Robson effects this by an incision, Sir M. Mackenzie by a puncture. The former method is always attended with hæmorrhage, which in many cases is very profuse; the latter is bloodless, because the pressure produced by the cannula plugs any vessel that may have been wounded, except in those rare cases in which a large vessel in the interior of the cyst becomes injured, and in these no serious result need be feared, because but little, if any, escape of blood can take place externally by the side of the cannula, and the amount of flow internally is limited by the size of the cyst, even if pressure and ice-bags have failed to check it previously. In order to destroy the secreting surface, Mr. Mayo Robson advocates scraping the wall of the cyst sufficiently to separate all colloid material, and subsequently packing the cyst with zinc lotion and lint. Sir M. Mackenzie recommends a solution of perchloride of iron to be injected into the cavity, with the twofold object of checking the hæmorrhage from the cyst walls which follows the withdrawal of the fluid contents, and subsequently of producing sufficient inflammation to destroy the secreting surface. Bearing in mind the extreme vascularity of the cyst wall and the delicate structure of the vessels, I consider Mr. Robson's method of destroying the secreting surface to be highly objectionable, even if not positively dangerous. The steps of the operation recommended by Mr. Robson cannot be performed without producing hæmorrhage, which is always liable to be profuse; the object of Sir M. Mackenzie's treatment, on the contrary, is throughout to prevent hæmorrhage. Holding the views above stated with regard to the method of treatment advocated by Mr. Mayo Robson, I am unable to consider it either simple, safe, or certain.

Having been accustomed myself to treat cases of cystic goitre by Sir M. Mackenzie's method and to see the same course adopted by others, I was unaware of the methods of dealing with this disease advocated in the last editions of the leading works on surgery, having had no occasion to refer to them on this subject, being well satisfied with the plan I employed, and knowing how disastrous many of the older modes of treatment had proved. It was not until my attention was called to the matter by the case referred to, published by Dr. Vachell last year, that I looked at the most recent English and American works on surgery to see what treatment they recommended. Had I done so, being ignorant of the best course to pursue, I confess that when I had finished such study I should have been a sadder but not a wiser man, because no assistance would have been obtained, although the five leading works had been consulted. I should have found that no two authors agreed upon what is the best method to adopt, and that, in fact, all recommended a different proceeding. My efforts to obtain advice would have shown me that Messrs. Holmes and Hulke advocated Sir Morell's Mackenzie's method; that Mr. Bryant recommended puncture as the first means to be adopted; that Mr. Erichsen advised the fluid contents to be drawn off by tapping, and an endeavour made to 
cause the cyst to close by inducing inflammation by means of an injection of iodine; that Professor Gross stated that six methods of treatment were at the command of the surgeon, all more or less serviceable, but not one entirely free from danger; and that Professor Agnew apparently did not consider the subject of sufficient importance to necessitate a separate paragraph being given to it, ho having enumerated the various methods of treatment applicable to the several forms of goitre in one list, and in such a manner that only a person possessing a full and accurate knowledge of the subject could distinguish which method was suitable to a particular form of the disense. A closer examination of the treatment recommended would have shown me that in two of the three English works injection of tincture of iodine was favourably spoken of, but under different conditions, Mr. Erichsen having advised the cyst to be emptied before the iodine was injected, whereas Mr. Bryant advocated the injection of tincture of iodine and alcohol in equal parts, without any allusion to the preliminary measures recommended by Mr. Erichsen; and that Professor Gross, on the other hand, whilst stating that injections of iodine occasionally succeed, went on to say that the cperation was liable to be followed by severe inflammation, eventuating in rapid reaccumulation and was in the main less certuin than the use of a seton, whereas Mr. Bryant stated that this latter method was dangerous, and should only be adopted when simple means were unavailing and further interference necessary. With such diversity of opinion it would have been extremely difficult for me to come to a couclusion as to what was the best course to adopt, especially as the weight of opinion was against the treatment which seemed most rational, and 1 consider that had I seleeted an operation which necessitated the exposure of my patient to considerable risk, the responsibility for the course adopted would have rested on the shoulders of most of the authors of the surgical works which deal with this disease, rather than on my own.

Considering the frequency with which cases of cystic goitre are mer with, and the large number of deaths which have followed the attempts to effect a cure, I maintain that the treatment of this disease demands more serious attention than surgeons have hitherto accorded it.

\section{LAPAROTOMY FOR TUBERCULAR PERITONITIS.}

TWO CASES OF IAPAROTOMY FOR ASCITES CAUSED BY TURERCULAR PERITONITIS; BOTH CURED.

\section{BY JOHN HOMANS, M.D.,}

SURGEON TO THE MASSACHUSETTS GENERAL HOSPITAL, AND HARYARD UNIVERSTTY LECTURER ON THE DIAGNOSIS AND TREATMENT

$$
\text { OF OVARIAN TUMOURS. }
$$

IN The LANCET for Nov. 5th, 1887, is an excellent report of a very interesting discussion at the Clinical Society of London. Mr. Lawford Knaggs does me the honour to refer to a case of mine in connexion with one of Sir Spencer Wells's, albeit I figure as Dr. "Hornan." In tracing the subsequent history of my patients after they have left my hands I have found another case, and both seem to me worth publishing.

In June, 1884, Dr. Tower of South Weymouth, sent me a pale, thin, emaciated girl, twenty-one years old, with a large abdomen full of fluid. Her weight was $90 \mathrm{lb}$. There was no swelling of the feet. The catamenia had been absent four months, and the girl seemed near her end from general failure of her constitution. I opened the abdomen on June 19th, 1884, and removed about $12 \mathrm{lb}$. of ascitic fluid. The stomach, apparently distended by fluid, I found as a large tumour in the upper portion of the abdomen; it was about ten inches in diameter Owing to lymph and adhesions the spleen and liver could not be felt. Much lymph covered the peritoneum in flakes and layers, and lay upon the abdominal viscera The peritoneum was studded over with little white deposits of tubercles, and nodules were also seen. A piece of the peritoneum was cut out and sent to a pathologist, who reported that the deposits were tubercles, but that the bacillus had not been found. In short, the disease was what we have always called tubercular peritonitis. The abdomen was sponged out as thoroughly as could be done without disturbing the viscera more than was thought prudent, and the wound was closed without the insertion of a drainage tube And here let me say that I have never been able to establish an artificial drain in a case of ascites by the insertion of a drainage tube. I have tried to do this again and again, but have never succeeded. When Nature means to cure the patient by drainage she reopens the wound. The suture were removed on the eighth day, and the wound was found united. In about ten days more the abdomen had become distended with fluid, and on July 7th the scar opened and the fluid ran out, and continued to do so. On July 12th the patient went home in good spirits. The ascitic fluid continued to run out, but was not offensive, and I suppose not septic. In November, 1884, she had gained considerable flesh, and had a good appetice. In February, 1885, the catamenia, which had been absent a year, reappeared, and the ascitic discharge was much diminished. In May the amount of discharge was very slight and she was able to walk out of doors; she was married in June. I saw her in January, $1886^{\circ}$; sh $\theta$ was fat and strong and was doing her housework; her weight was $121 \mathrm{lb}$. I received a visit from my patient again in November, 1886 ; she then weighed $130 \mathrm{lb}$, and was quite strong. She had never been pregnant. There was a slight sinus, which discharged about a drachm of pus a day. The case seemed to me at the time, and seems to $m \in$ now, a wonderful recovery.

The second case is one in which the patient was not emaciated, and in which I had expected to find an ovarian or parovarian cyst. The patient was a school-girl, seventeen years old, of healthy appearance. Her complexion was pale. Her umbilical girth was thirty-four inches, and thought I felt a very faint aortic impulse. The catamenia were normal. She was sent to me in April, 1887, by Dr. Kingsbury, of Lyme, New Hampshire. The abdomen had begun to enlarge twelve months before, and the process of enlargement had been slow. On opening the peritoneum on April 20th, 1887, ascitic fluid ran out, and on further investigation the whole abdominal cavity was found to be studded with small hard whitish nodules. They were on the uterus, intestines, and parietal surface of the peritoneum. both ovaries, and Fallopian tubes. One tube thickly studded with tubercles, with its corresponding ovary, was removed. The peritoneal cavity was carefully sponged out, but the ascitic fluid seemed to reaccumulate so fast that a glase drainage tube was put in and the abdomen closed. The. specimens were sent to Professor Fitz, who reported that the deposits were tubercular. Only about an ounce of fluid in twenty-four hours was obtained from the drainage tube, and on the third day it was removed. The patient went home at the end of three weeks. On December 28th, 1887, eight months after the operation, I had a letter from Dr. Kingsbury, in which he says that the abdomen is no larger than natural; that she has no fever, pain, tenderness, or unpleasant sensations in the abdomen; and that her appetite is good. She is not very strong, but can endure more than before the operation; she does some light work in the family and gives lessons in music.

The second case is not as remarkable as the first, but perhaps both will aid in the study of the treatment of tubercular peritonitis by laparotomy.

\section{NOTE ON THREE CASES OF OBLITERATIVE ARTERITIS.}

BY W. B. HADDEN, M.D.LoND., M.R.C.P. assigTaNT PHYSIClay aND DEMONSTRaTOR OF MORBID ANaTOMY TO ST. THOMAS'S HOSPITAL, ASSISTANT PHYSICLAN TO THE HOSPITAL FOR SICK CHITDREN.

THE disease known as "obliterative arteritis" is by no means common, and its pathological nature is still uncertain The case which I am about to describe is unfortunately incomplete. I only saw the patient once, but I satisfied myself that the affection was identical with that described by Mr. Pearce Gould and by myself in vol. xvii. of the Clinical Society's Transactions:

The patient was a fairly robust young man, aged ninteen, a rug-sorter by trade. He came to me as an out-patient at St. Thomas's Hospital on December 10th, 1887, complaining

1 This case may be found briefiy reported in a little book entitled. "Three hundred and eighty-four Laparotcmies for various Disezses," by John Homans, Boston, 1887. 